\title{
Um estudo da dinâmica interna para um problema com Rigidez Não Linear
}

\section{Célia Aparecida dos Reis ${ }^{1}$,}

Faculdade de Ciências, Departamento de Matemática, Unesp, Bauru, SP

Luis Antonio S. Vasconcellos ${ }^{2}$

Faculdade de Ciências, Departamento de Matemática, Unesp, Bauru, SP

\section{Luttgardes de Oliveira Neto ${ }^{3}$}

Faculdade de Engenharia, Departamento de E. Civil e Ambiental, Unesp, Bauru, SP

\begin{abstract}
Resumo. A equação de Duffing nas suas várias formas pode ser utilizada para descrever diversos sistemas não lineares. Embora a maioria dos sistemas físicos não possa ser descrita com precisão desta forma, para uma ampla gama de condições operacionais tais como a frequência e amplitude de excitação, em muitos casos, é possível a utilização desta equação como uma descrição aproximada, de modo que seu comportamento possa ser estudado qualitativamente. Neste trabalho considera-se a análise das deflexões grandes de uma viga com rigidez não linear, cujo modelo matemático pode ser descrito por uma equação de Duffing. A linearização exata no espaço de estados é o método proposto para análise desta dinâmica não linear. Apresenta-se a análise da dinâmica interna e da estabilidade assintótica local em complementação a trabalhos publicados no ano de 2014, levando-se em conta os pontos críticos não nulos deste sistema não linear. Finalmente, exemplos numéricos são apresentados.

Palavras-chave. Equação de Duffing, Deflexão de vigas, Linearização entrada-saída, Estabilidade assintótica, Dinâmica zero.
\end{abstract}

\section{Introdução}

Os projetos de grandes estruturas que envolvem dados de difícil definição têm utilizado cada vez mais o recurso de monitoração durante a sua construção para tomadas de decisões. Nesses momentos, as comparações entre resultados da monitoração com os obtidos de modelos matemáticos têm permitido a continuidade na execução dessas obras com maior controle e qualidade [1]. A análise estrutural desempenha um papel importante no desenvolvimento do projeto de tais estruturas e modelos matemáticos cada vez mais sofisticados propiciam análises inimagináveis até alguns anos atrás, fornecendo subsídios para análises paramétricas e permitindo simulações de etapas construtivas com o nível de detalhamento desejado pelos projetistas [3].

Como exemplos de problemas de vibrações não lineares podem ser citados as vibrações de vigas, de cabos, de um pêndulo, de alguns isoladores, de circuitos elétricos,

${ }^{1}$ celia@fc.unesp.br

2 toninho@fc.unesp.br

3 lutt@feb.unesp.br 
cujos modelos matemáticos são muitas vezes descritos por equações do tipo da Equação de Duffing [4 - 5].

Como o movimento do primeiro modo de vibração de uma viga bi-apoiada nas extremidades, com rigidez não linear e de comprimento 1, pode ser modelado matematicamente por uma equação de Duffing, a metodologia abordada nestas notas será a linearização entrada-saída, aplicada nas vibrações grandes de uma viga bi-apoiada, com rigidez não linear.

O objetivo é decompor a dinâmica do sistema não linear da viga bi-apoiada na chamada forma normal, isto é, em uma parte externa linear (entrada-saída) e uma parte interna não linear e não observável. Para tal, define-se um novo conjunto de estados, definidos a partir da saída e suas derivadas. Desta forma, prova-se a existência de um difeomorfismo, o qual transforma o sistema não linear em outro linear de menor grau, relacionando a entrada e a saída, e em uma parte que pode ser tanto linear quanto não linear, que representa os estados não observáveis e que fornece a dinâmica interna do sistema original ([8], [9]).

O conhecimento da dinâmica interna é importante, pois, a partir de seu equacionamento, torna-se possível a análise da dinâmica zero, a qual pode ser utilizada para análise de estabilidade assintótica da dinâmica não linear em malha fechada; assim avalia-se se a linearização por realimentação pode ser usada (qualquer sistema em malha fechada tem que ser estável) ([7], [16]).

Neste trabalho apresenta-se a análise da dinâmica interna e da estabilidade assintótica do modelo matemático da viga bi-apoiada, além da utilização da linearização entrada-saída para encontrar uma lei de controle não linear que possibilita o controle das vibrações, em complementação aos resultados obtidos em [17], [18] e [19]. Finalmente, exemplos numéricos são apresentados.

Este trabalho encontra-se organizado da seguinte forma: na seção 2 apresenta-se a linearização entrada-saída para a viga bi-apoiada. Na seção 3 apresenta-se o estudo da dinâmica zero e da estabilidade assintótica local e simulações e na seção 4 as conclusões e sugestões de trabalhos futuros.

\section{O Modelo Matemático Utilizado e a Linearização Entrada-Saída}

Considere o oscilador de Duffing com uma força externa e um termo cúbico, dado por:

$$
\ddot{x}+2 \xi \dot{x}+\alpha x+\not x^{3}=F \cos \Omega t
$$

sendo que $x, \xi, \alpha, \gamma, f, \Omega$ são respectivamente o deslocamento, o fator de amortecimento, parâmetro de rigidez, parâmetro de rigidez não linear, amplitude de excitação e frequência de excitação, conforme [10], [12].

Em (1), quando $\xi=0$ e $\alpha=1$ esta equação descreve o movimento do primeiro modo de vibração de uma viga bi-apoiada nas extremidades, de comprimento $l$, como na Figura 1, considerando o suporte do lado esquerdo fixo e o da direita livre para deslizar com o objetivo de evitar alongamento no plano da viga quando esta vibrar ([10]).

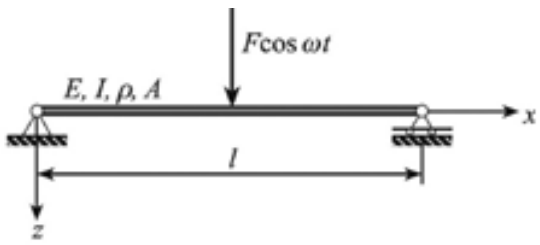

Figura 1: Vibrações de uma viga com elasticidade não linear. 
De acordo com [10], uma equação que representa o movimento do primeiro modo de vibração da viga, em termos de $L$ é dada por:

$$
m \ddot{q}+k_{1} q-k_{3} q^{3}=F \cos \omega t
$$

sendo:

$$
m=\frac{\rho A l}{2} \quad k_{1}=\frac{E I \pi^{4}}{\left(2 L^{3}\right)} \quad k_{3}=\frac{-3 \pi^{6} E I}{\left(16 L^{5}\right)}
$$

A função amplitude de deslocamento tem a forma:

$$
\frac{L}{l}=\frac{1}{2}\left(1+\left(1-\left(\frac{q \pi}{2 l}\right)^{2}\right)^{\frac{1}{2}}\right)
$$

Após a substituição de (3) em (2) seguido do desprezo de potências de $q$ maiores do que três, o termo cúbico da equação (2) é positivo, e daí a viga apresenta rigidez para grandes deflexões.

Conforme [10], a equação (2) pode ser escrita como:

$$
\ddot{\tilde{y}}+\tilde{y}+\tilde{y}^{3}=\tilde{F} \cos \Omega \tilde{t}
$$

sendo $\tilde{y}=\frac{q}{l}, \tilde{t}=w_{n} t, \Omega=\frac{w}{w_{n}}, w_{n}=\sqrt{\frac{k_{l}}{m}}, \tilde{F}=\frac{F}{k_{l} l}, \gamma=\frac{l^{2} k_{3}}{k_{l}}$.

A equação (4) pode ser escrita na forma:

sendo:

$$
\begin{aligned}
& \dot{x}=f(x)+g(x) \tilde{u}(t) \\
& y=h(x)
\end{aligned}
$$

$$
f(x)=\left[\begin{array}{c}
x_{2} \\
-x_{1}-\gamma x_{1}^{3}
\end{array}\right], \quad g(x)=\left[\begin{array}{l}
0 \\
1
\end{array}\right], \quad \tilde{u}(\tilde{t})=\tilde{F} \cos \Omega \tilde{t} \text { e } \quad y(x)=a x_{1}+b x_{2}, b \neq 0 .
$$

Prova-se que o grau relativo do sistema (5) é $r=1$, sendo possível a análise da dinâmica interna e da dinâmica zero do sistema (5) ([7], [16], [17], [19]). Para tal, torna-se necessário a construção de um difeomorfismo $\phi(x)=[\mu, \psi]$ sendo $y=h(t)=a x_{1}+b x_{2}$ e $\psi$ uma solução do conjunto de equações diferenciais parciais (ou EDP) $\nabla \psi \cdot g(x)=0$ ou $\frac{\partial \psi(x)}{\partial x_{2}}=0$, de acordo com [7] e [16].

Conforme [19] a função $\phi(x)$ é dada por:

$$
\phi=\left[a x_{1}+b x_{2}, \psi\right]
$$

sendo $\psi$ a solução da $\operatorname{EDP} \frac{\partial \psi(x)}{\partial x_{2}}=0$, como em (7):

$$
\psi(x)=c x_{1} .
$$

Prova-se que $\phi(x)$ em (6) é um difeomorfismo global, sendo o vetor de estados $\left(x_{1}, x_{2}\right)$ :

$$
x_{1}=\frac{1}{c} \psi \quad \mathrm{e} \quad x_{2}=\frac{1}{b} \mu-\frac{a}{b c} \psi .
$$

Além disso, $\phi(x)$ tem inversa: 


$$
\phi^{-1}(\mu, \psi)=\left(\frac{1}{c} \psi, \frac{1}{b} \mu-\frac{a}{b c} \psi\right)
$$

A partir do conjunto de estados definidos em (8) o sistema (5) tem a forma normal ([7], [16], [17], [19]):

$$
\begin{aligned}
& \dot{\mu}=\left(\frac{a}{b}\right) \mu-\frac{1}{c}\left(\frac{a^{2}}{b}+b\right) \psi-\frac{\gamma b}{c^{3}} \psi^{3}+b \tilde{u}(\tilde{t}) \\
& \dot{\psi}=\frac{c}{b} \mu-\frac{a}{b} \psi \\
& \tilde{y}=\mu
\end{aligned}
$$

A dinâmica não linear dada por (9) relaciona entrada e saída, correspondendo à parte externa do sistema, enquanto que a dinâmica (9-a) corresponde a parte interna da dinâmica, não dependente da entrada $u(t)$. Portanto, o sistema (9-a) corresponde à parte não observável e não controlável do sistema. De acordo com [17] e [19], a lei de controle não linear de equação (10) transforma a parte externa do sistema não linear (9) - (9-a), em uma dinâmica linear:

$$
\tilde{u}(\tilde{t})=\frac{1}{b}\left\{\left(-\frac{a}{b}\right) \mu+\frac{1}{c}\left(\frac{a^{2}}{b}+b\right) \psi+\frac{\gamma b}{c^{3}} \psi^{3}+v\right\} .
$$

\section{A análise da Dinâmica Zero do Sistema e da Estabilidade Assintótica}

De (5), e como $\alpha=1 \neq 0$, tem-se que a dinâmica não linear tem três pontos críticos, a saber:

$$
P_{1}=(0,0), \quad P_{2}=\left(\sqrt{\frac{-1}{\gamma}}, 0\right) \text { e } \quad P_{3}=\left(-\sqrt{\frac{-1}{\gamma}}, 0\right)
$$

se $\gamma<0$ e existe um único ponto crítico, a saber, $(0,0)$, se $\gamma>0$.

De (5) ou (9) - (9-a), tem-se a matriz da contraparte linear possui dois autovalores distintos e imaginários puros. Isso significa que $(0,0)$ é um centro estável para a contra-parte linear da dinâmica (5) ou (9) - (9-a). Desta forma, considerando-se a dinâmica não linear (5) ou (9) - (9-a), esse ponto crítico pode ser um centro ou um ponto em espiral, assintoticamente estável, ou estável, mas não assintoticamente estável ou instável. Desta forma, não é possível estabelecer uma conclusão a respeito da estabilidade assintótica do ponto crítico $(0,0)$ e nem de sua natureza.

Em [17] e [19] verificou-se, pela análise da dinâmica zero, a estabilidade assintótica da dinâmica não linear em torno da origem. Neste trabalho, a análise será efetuada em torno do ponto $P_{2}$. Considerando este ponto e a mudança de variáveis dada por:

$$
\mu_{1}=\mu-\sqrt{\frac{-1}{\gamma}} \text { e } \psi_{1}=\psi
$$

prova-se que a dinâmica zero, dada quando $y(t)=0$ para todo $t>0$, pode ser escrita na forma:

$$
\dot{\psi_{1}}=-\frac{a}{b} \psi, \quad y=0
$$


cuja resposta no tempo é dada pela expressão $\psi_{1}(\tilde{t})=e^{\left(-\frac{a}{b}\right) \tilde{t}}$.

Assim, a dinâmica zero (13) é assintoticamente estável quando $a, b<0$ ou $a, b>0$, em torno do ponto, coincidindo com os resultados obtidos em torno do ponto $P_{l}$ ([16], [19]). De fato, considerando-se o controle não linear:

$$
\tilde{u}(t)=\left(\frac{-a-k_{o}}{b}\right) \mu_{1}+\left(-\frac{1}{c}\right) \psi_{1}+\left(-\frac{\gamma}{c^{3}}\right) \psi_{1}^{3}+\sqrt{-\frac{1}{\gamma}}\left(\frac{a-k_{o}}{b}\right)
$$

para $v=-k_{o} y=-k_{o} a x_{1}-k_{o} b x_{2}$, e $k_{o}$ escolhido de forma conveniente tal que $p(\lambda)=\lambda+k_{o}$ tenha raízes negativas ([16]), torna a malha fechada da dinâmica (5) assintoticamente estável em torno do ponto $P_{2}$.

Observa-se que a análise é análoga para o ponto $P_{3}$ e também a vantagem da linearização entrada-saída para o estudo da estabilidade assintótica da dinâmica não linear. Pelo método direto de Lyapunov não é possível efetuar a análise da estabilidade em torno de $P_{2}$. Mediante o uso da estabilidade assintótica da dinâmica zero foi possível determinar a estabilidade assintótica da dinâmica não linear não só em torno da origem como dos outros dois pontos.

A Figura 2 mostra o efeito na resposta do sistema não linear (5), da lei de controle (14) de acordo com os valores dos parâmetros adotados na Tabela 1.

Tabela 1. Valores de parâmetros

\begin{tabular}{|c|c|c|c|c|c|c|c|c|c|c|c|}
\hline Parâmetro & $\boldsymbol{\alpha}$ & $\boldsymbol{\Omega}$ & $\boldsymbol{\gamma}$ & $\boldsymbol{F}$ & $\boldsymbol{a}$ & $\boldsymbol{b}$ & $\boldsymbol{c}$ & $\boldsymbol{k o}$ & $\boldsymbol{L}$ & $\boldsymbol{L}$ & $\boldsymbol{I}$ \\
\hline Caso 1 & 1 & 0,5 & 5 & 0,3 & 0,1 & 1 & 1 & 1 & 400 & 400 & 4616 \\
\hline Caso 2 & 1 & 0,5 & 5 & 0,3 & 1 & 0,1 & 1 & 1 & 400 & 400 & 4616 \\
\hline Caso 3 & 1 & 0,5 & 5 & 0,3 & 1 & 1 & 0,1 & 1 & 400 & 400 & 4616 \\
\hline
\end{tabular}

Considerando um exemplo prático com dimensões realistas de uma viga metálica, podese avaliar o comportamento da viga em função dos dados de controle. Para uma resposta dinâmica inicial, avalia-se a influência dos parâmetros $a, b$ e $c$ no problema. Assim os dados de três situações estão dispostos na Tabela 1, mantendo constante o perfil metálico, e com o peso distribuído apenas o seu peso próprio.

Considera-se um intervalo de tempo de 0 a 10.000 s, na Figura 2, para que se perceba o comportamento assintoticamente estável do sistema, já que a rigidez nesse caso é grande e o sistema não apresenta amortecimento. Verifica-se na Figura 2, o quanto é eficiente o controle dado por (14), já que este leva a resposta para a origem em um tempo muito pequeno. As informações relativas ao Módulo de Young do aço, peso específico e força encontram-se em [19]. O comprimento $l$ da viga está mensurado em $\mathrm{cm}$.
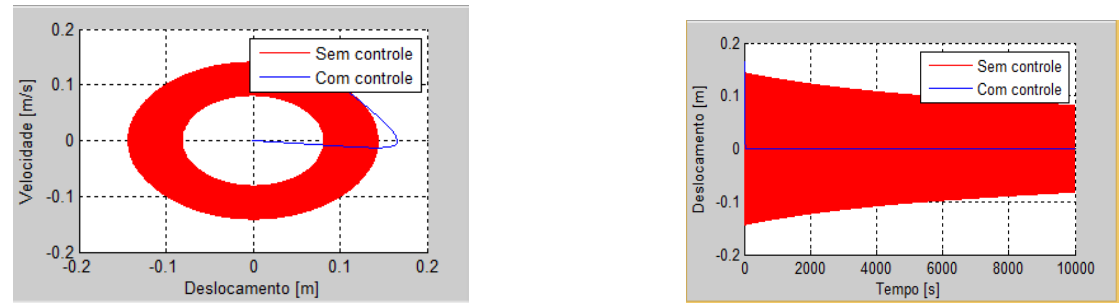

(a) Caso 1: $a=0,1, b=1$ e $c=1$ 

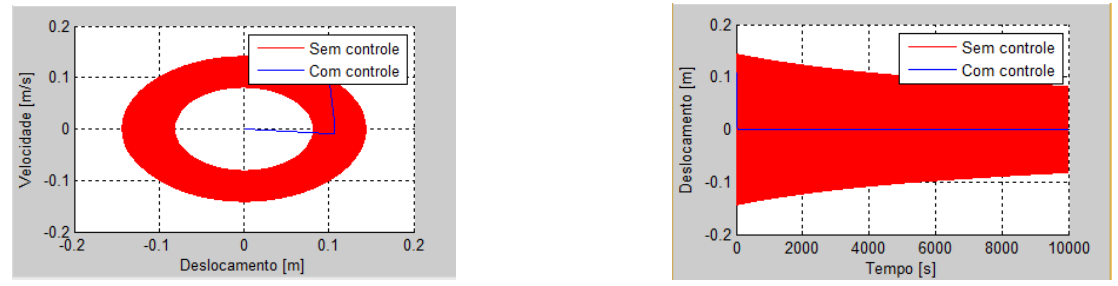

(b) (b) Caso 2: $a=1, b=0,1$ e $c=1$
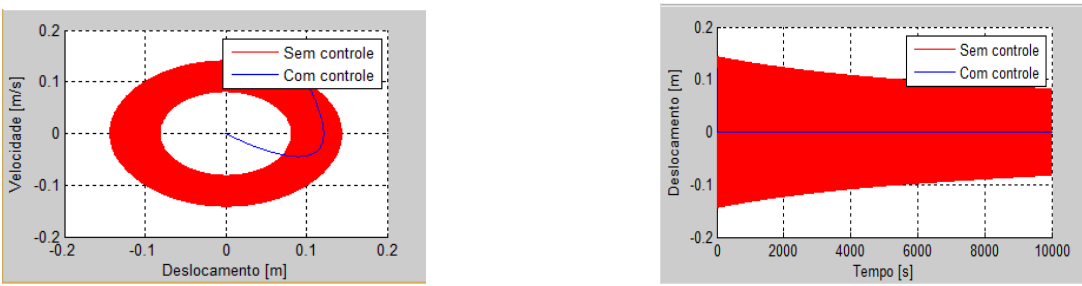

(c) Caso 3: $a=1, b=1$ e $c=0.1$

Figura 2 - Retrato de fase e histórico do deslocamento no tempo para $u(t)=F \cos \Omega t$.

\section{Conclusões}

Neste trabalho considerou-se a análise das deflexões grandes de uma viga com rigidez não linear, cujo modelo matemático utilizado é descrito por uma equação de Duffing. Mediante a análise da dinâmica interna do sistema, provou-se que a dinâmica não linear é assintóticamente estável não só em torno da origem, mas também em torno dos pontos fora da origem.

Introduziu-se um controle não linear (linearização por realimentação) que possibilitou a estabilização deste sistema em um intervalo de tempo bastante pequeno (realimentação de estados externa).

Simulações foram efetuadas, com e sem o controle via linearização por realimentação, onde foram feitas variações da frequência de oscilações e levando-se em conta a análise da esbelteza da viga.

Para trabalhos futuros, deseja-se analisar a primeira deflexão da viga para uma carga constante, além de uma análise das vibrações resultantes dessa carga, após um intervalo de tempo. Além disso, deseja-se efetuar uma análise global do sistema, com e sem controle, com variações dos demais parâmetros, além de avaliar a ação de uma carga, para uma viga esbelta, a ponto de levar a mesma a uma ruptura.

\section{Referências}

[1] R.D. Copetti, D. Migotto, D. R. Tolfo, Sobre a Resposta Dinâmica de uma Viga com Amortecimento, Mecânica Computacional, Vol. XXIX, págs.4247-4254,Argentina, 2010.

[2] N. Fiedler-Ferrara, C. P.C. Prado, Caos - Uma introdução, Edgar Blucher, S.P., 2009

[3] P. B. Fusco, "Estruturas de Concreto", Editora Guanabara, 1991.

[4] J. Ginsberg, "Mechanical and Structural Vibrations", John Wiley, 2002.

[5] D. Gorman, "Free Vibration Analysis of Beams and Shafts", John Wiley, 1975. 
[6] M. Gürgöze, H. Erol, "Dynamic response of a viscously damped cantilever with a viscous end condition", Journal of Sound and Vibration, 298:132"U153, 2006.

[7] A. Isidori, Nonlinear Control Systems, 3ed., Springer-Verlag, Roma, 1995.

[8] M. K. Jain, S. R. K. Iyengar, R. K. Jain, "Numerical Methods for Scientific and Engineering Computation", Ed. John Wiley \& Sons, 1985.

[9] S. G. Kelly, “Advanced Vibration Analysis”, John Wiley, 1 st edition, 2006.

[10] I. Kovacic,, M. J. Brennan- The Duffing Equation Nonlinear Oscillators and Their Behavior, Wiley \& Sons, 2011.

[11] S. Naguleswaran, "Vibration of an Euler-Bernoulli beam on elastic end supports and with up to three step changes in cross-section", International Journal of Mechanical Sciences, 44:2541-2555, 2002.

[12] S. S. Raos, Mechanical Vibrations, Pearson Prentice Hall, New Jersey, 2004.

[13] C. A. Reis et al., Asymptotic Stabilization and Internal Dynamics of a Simplified Model of a Maglev System, 22nd International Congress of Mechanical Engineering (COBEM), November 3-7, Ribeirão Preto, SP, Brazil, 2013.

[14] C. A. Reis et al., The Input-State Linearization Of A Maglev Vehicle Type, 22nd International Congress of Mechanical Engineering (COBEM), Ribeirão Preto-SP, Brazil, 2013.

[15] Reis, C. A.; Colón, D. ; Balthazar, J. M. ; Rosa, S. R. F. ; Rocha, R. T. (2014). O Comportamento de Sistemas Mecatrônicos usando Ferramentas da Dinâmica Zero. In: Airam Sausen; Paulo Sausen; Sandro Sawicki.. (Org.). Coleção Modelagem Matemática Aplicada à Resolução de Problemas nas Engenharias: 1ed.Ijuí: Editora Unijuí da Univ. Regional do Noroeste do Estado do Rio Grande do Sul, 2014, v.3, p.169-194.

[16] C. A Reis, J. M. Balthazar; D. Colón; S. R. F. Rosa; R. T. Rocha; B. R. Pontes Junior Análise das Vibrações Forçadas de um Oscilador de Duffing via Linearização Exata. In: VIII Congresso Nacional de Engenharia Mecânica (CONEM 2014), 2014, Uberlândia.

[17] G. V. M. Silva, Controlo Não Linear, Escola Superior de Tecnologia Setúbal, Lisboa, 2003.

[18] J. Slotine and W. LI, Applied Nonlinear Control. New Jersey: Prentice Hall, 1991.

[19]L.A.S Vasconcellos, C. A. Reis, L.O Neto, “Análise das Deflexões Grandes de uma Viga Com Rigidez Não Linear Utilizando a Linearização Exata a Realimentação, Anais do XXXV Congresso Nacional de Matemática Aplicada e Computacional, Natal, RN, Brasil, 2014. 